\title{
FROM LIMIT EQUATIONS TO GENERAL EQUATIONS: AN ALTERNATIVE STRATEGY OF DEVELOPING EQUATIONS IN CHEMISTRY AND ITS DIFFERENT STAGES OF LEARNING
}

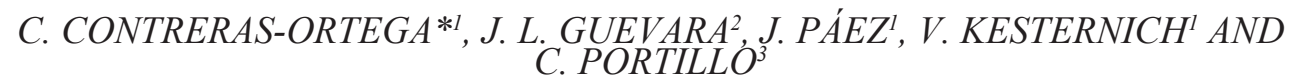 \\ ${ }^{\text {I} D e p a r t a m e n t o ~ d e ~ Q u i ́ m i c a, ~ F a c u l t a d ~ d e ~ C i e n c i a s, ~ U n i v e r s i d a d ~ C a t o ́ l i c a ~ d e l ~ N o r t e, ~}$ \\ Casilla 1280, Antofagasta, Chile. \\ ${ }^{2}$ Departamento de Biología y Química, Facultad de Ciencias Básicas, Universidad Católica del Maule, Casilla 617, Talca, Chile \\ ${ }_{3}^{3}$ Departamento de Ingeniería Eléctrica, Universidad de Antofagasta, Casilla 170
}

(Received: August 16, 2012 - Accepted: July 11, 2013)

\begin{abstract}
The scope of limit equations and their role as starting points to deduce general equations are discussed. Consequently, a general strategy is proposed to go systematically from limit equations to general ones. On the basis of this strategy, the most common general equations in chemistry, like the equation of state for gases, the chemical potential of gases and the equation for the chemical equilibrium constant are illustrated. The method should help to emphasize students' need to think about the validity range of the equations they are learning and to illustrate the way science infers general equations from specific ones. Furthermore, it should allow explaining to students the several steps (introductory, medium and upper level) of their chemistry learning.
\end{abstract}

Key words: General Chemistry, Physical Chemistry, Thermodynamics, Ideal Gases, Real Gases, Equation of States, Chemical Potentials, Chemical Equilibrium Constants

\section{INTRODUCTION}

General Chemistry textbooks mostly teach equations strictly valid only for systems under limiting physical conditions ${ }^{1-3}$. A rather recently article has also called the attention on this fact and on how it limits the analysis of more general situations, allowing propagations of misconceptions among students ${ }^{4}$.

Limiting conditions are normally defined in terms of physical or chemical parameters at their limiting values, such as infinitely dilute concentrations, infinitely high temperatures or low pressures, etc. Examples of these limit equations are the well known concentration-based equations for chemical equilibrium constants, Nernst's equation for electrochemical cells, Raoult's and Henry's laws for solution vapor pressures, colligative properties of solutions, and the widely used ideal gas equation $P V=n R T$.

Several questions arise regarding limit equations: Why do they appear? What part of Nature do they explain? How are situations other than the limiting ones explained? These questions are not often discussed in first-year chemistry textbooks, but their importance in building students' understanding of the limitations of models and algorithms can not be overstated. Specific answers are given in upper level chemistry courses, where concepts like activity and activity coefficients are introduced.

The aim of this article is to answer the questions above in a general way to better understand the real scope of limit equations and their role in deriving equations for real systems under any condition. Consequently, the article proposes a general strategy to go systematically from limit equations to general ones. This article also aims at illustrating the way science infers general equations from specific ones. We believe that this methodology is not consciously perceived by students in the long transit from the limit equations of general chemistry courses to the general ones learned in their last physical chemistry courses. Generally, textbooks do not make this role of science explicit to students either, although the value of the limit laws in science has been mentioned earlier ${ }^{5}$

\section{METHOLOGY}

\subsection{A Scientific Route to Understanding}

Limit equations are mathematical formulations of laws describing the regular behaviors of systems under limiting physical conditions. It has been possible to observe these regularities in the laboratory because, as we approach these limiting conditions, matter interactions become simpler and can be explained using readily observable parameters. Nevertheless, science must look for general equations capable of explaining the whole behavior of nature. In this regard, limiting physical situations must also be explained by these general equations. Then, limit equations must be particular forms of these general equations.
One of the ways science seeks the general equations of nature is as follows. First, scientists simplify the realities they are trying to understand by progressively making the experiment that represents them more and more simply until they start observing regularities; they begin to understand those realities. Regularities are then expressed as laws and encoded as mathematical formulas. The limiting systems are simplified experimental systems. Second, these limiting real situations are taken as models (experimental models) to image idealized experimental situations, which either eliminate the boundary restrictions of limiting real systems or restrict reality to the boundaries where it can be understood. Because of that, these systems are considered as ideal ones. Thus, limit laws and equations are raised to the category of general laws; they are now capable of describing an idealized version of nature in its whole range of existence. These ideal systems, and their laws and equations, are then submitted to the formalism of general equations and new equations are deduced for them. The final step is to modify these fictional general equations to obtain real general equations capable of giving account of the complete reality. Scheme 1 illustrates this procedure for a particular system.

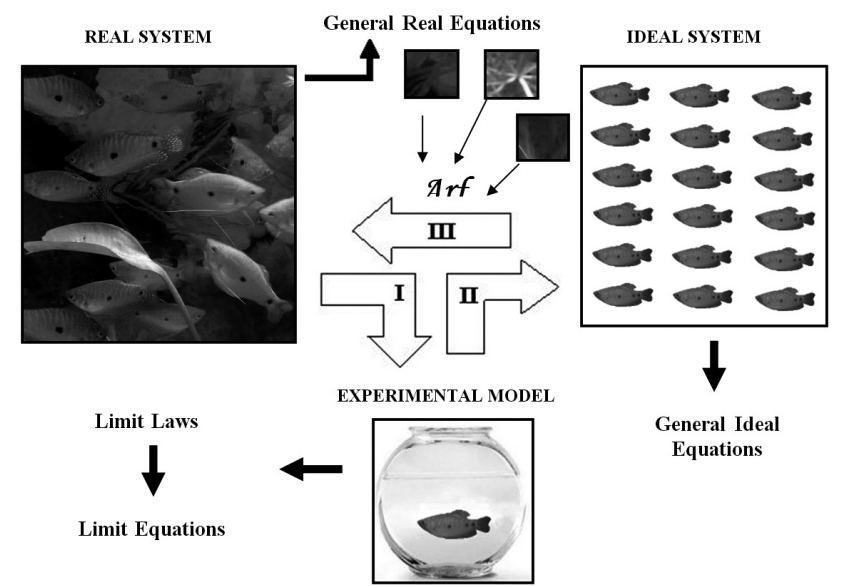

Scheme 1. The scientific route to understanding. Step I: simplifying the real system to obtain equations for some reality we can understand; limit equations for a limiting real system. Step II: building-up an ideal system to obtain general equations for a fictional (model) system; generalizing the limiting situation. Step III: back to reality to obtain general equations for the general real system; general ideal equations are modified by functions, $A_{r p}$, containing all the relevant information about the reality ignored by the ideal system. 


\subsection{An Example}

Let's apply above scheme to gases, real gases, the only ones available to us. We start by concentrating our attention on gases under limiting physical conditions, which we will name limiting real gases, the only ones we understand well at this stage. Then, we will first deduce all the equations (or main) for these limiting (real) gases; equations that will be valid in reality only under limiting physical conditions. From these equations we will then infer all the ideal equations by just ruling out from the limiting equations their boundaries restrictions. Thus inferred, these equations will cover a hypothetical universe because equations that are valid in reality only under limiting conditions will now be valid at any conditions. Therefore, they will be equations for an ideal universe, ideal gases, and hence (general) ideal equations. We will finally infer all real general equations from above ideal equations, where no physical restrictions exist, by bringing them to reality. We will do this by introducing into these equations all aspects ignored by the ideal equations by mean of appropriate functions, that we will name Approximation to Reality Functions, $A_{\text {. }}$. Thus, we will arrive to equation for general real gases. In summary, we will deduce all general equations from their corresponding limiting equations by using ideal equations as a methodological bridge between them. Table
1 shows ours results. Following the footnote of Table 1, primed equation numbers (') in the text will refer to equations given in the table. Limit equations and parameter tendencies (e.g. $P \rightarrow 0, T \rightarrow \infty$ ) in both the table and the text have the meaning we discussed in a previous article ${ }^{6}$

Usually equations for one step (for one column in Table 1) are not necessarily deduced after all equations for the previous step (for the previous column) are deduced. Neither the deductions of equations in one row of Table 1 are necessarily made one after the other. Thus, no matter what each equation represents, Eq 5' is commonly deduced following the route of Eqs 1'- 4'-5', while the deduction for Eq 8' follows the route of Eqs 1'- 4'-5'-8'; Eq 6' and $\mathrm{Eq}$ 9' are deduced from Eq 5' and Eq 8', respectively; and Eqs 2' and $3^{\prime}$ are deduced as particular cases of Eqs 8' and 9', respectively, ${ }^{5,9}$. We will now deduce all equations strictly following the sequence shown in Scheme 1 , the equations for each step corresponding to each column in Table 1 in the same sequence. We believe that this strategy should look more logical and didactical to students. The need of using a logical sequence in the development of Thermodynamic for its better understanding has already been claimed in another paper $^{10}$, though the authors do not make reference to any particular sequence.

Table 1. Limit, Ideal and General Equations for Gases.

\begin{tabular}{|c|c|c|c|}
\hline Limit Real Eqs, $E_{\text {lim }}$ & General Ideal Eqs, I & General Real Eqs, $\mathbb{R}$ & Approximation-to-reality functions, $\mathcal{A}_{r f}$ \\
\hline $\begin{array}{l}\left(1^{\prime}\right)(P V)_{\text {lim }}=\left(\Sigma_{i} n_{i}\right) R T \\
P \rightarrow 0, T \rightarrow \infty \text {, any } x_{i}\end{array}$ & $\begin{array}{l}\left(4^{\prime}\right)(P V)_{\text {ideal }}=\left(\sum_{i} n_{i}\right) R T \\
\quad \text { any T, } P \text { and } x_{i}\end{array}$ & $\begin{array}{c}\left(7^{\prime}\right)(P V)_{\text {real }}=(P V)_{\text {ideal }} x \mathcal{A}_{r f} \\
\text { any T, } P \text { and } x_{i}\end{array}$ & $\begin{array}{c}\left(11^{\prime}\right) A_{r f}=\left[1+A_{2}(T) P+A_{3}(T) P^{2}+A_{4}(T)\right. \\
\left.P^{3}+\cdots \cdot\right]\end{array}$ \\
\hline $\begin{array}{c}\left(2^{\prime}\right) \mu_{i, \text { lim }}=\mu_{i}^{o}(T)+R T \ln \left(P_{i} / P^{o}\right) \\
\quad \text { all } P_{i} \rightarrow 0, T \rightarrow \infty\end{array}$ & $\begin{array}{c}\left(5^{\prime}\right) \mu_{i, \text { ideal }}=\mu_{i}^{o}(T)+R T \ln \left(P_{i} / P^{o}\right) \\
\text { any } T \text { and } P_{i}\end{array}$ & $\begin{array}{l}\left(8^{\prime}\right) \mu_{i \text { real }}=\mu_{i \text { ideal }}+\mathcal{A}_{f f} \\
\quad \text { any T Tand } P_{i}=x_{i} P\end{array}$ & $\begin{array}{c}\left(12^{\prime}\right) \mathcal{A}_{r f}=R T \ln \chi_{i} \\
\chi_{i}=f_{i} / P_{i}\end{array}$ \\
\hline $\begin{array}{l}\text { (3') } K_{\text {lim }}=P_{C}{ }^{c} P_{D}^{d} / P_{A}^{a} P_{B}^{b} \\
\text { all } P_{i} \rightarrow 0, T \rightarrow \infty\end{array}$ & $\begin{array}{c}\left(6^{\prime}\right) K_{\text {ideal }}=P_{C}{ }^{c} P_{D}{ }^{d} / P_{A}^{a}{ }^{a} P_{B}^{b} \\
\text { any } T \text { and } P_{i}\end{array}$ & $\begin{array}{l}\left(9^{\prime}\right) K_{\text {real }}=K_{\text {ideal }} \times \mathcal{A}_{r f} \\
\text { any } T \text { and } P_{i}=x_{i} P\end{array}$ & $\left(13^{\prime}\right) \mathcal{A}_{r f}=\chi_{C}^{c} \chi_{D}^{d} \chi_{A}^{a} \chi_{B}^{b}$ \\
\hline $\mathcal{E}_{\text {lim }}$ & $I=\mathcal{E}_{\text {fim }}$ & $\left(10^{\prime}\right) \mathcal{R}=I x \mathcal{A}_{r f}$ or $\mathbb{R}=I+\mathcal{A}_{r f}$ & \\
\hline
\end{tabular}

Equation numbers are primed (') to distinguish them from those in the text. The first column shows thermodynamics equations for limiting real gases: equations for gases at extremely low pressures $(P \rightarrow 0)$ and high temperatures $(T \rightarrow \infty)$. The second column shows equations for ideal gases: same former equations but with no restrictions to pressure and temperature values. The third column shows equations for general real gases: same former ideal equations but modified by appropriate functions correcting their ideality, $A_{r f}$ with no restrictions to pressure and temperature values. The fourth column shows some $A_{r f}$ 's functions. The meanings of the terms in the last row as well as the deductions of all equations are given in the text.

\section{RESULTS AND DISCUSSIONS}

\subsection{Limiting Real Gases (the reality we can understand: a Fish in the} Bowl)

We can define a limiting real gas as one existing at limiting physical conditions. However, a more precise definition is needed and from a thermodynamics point of view.

The Equation of State of Limiting Real Gases. All gases obey the well known equation of state at certain temperature and pressure values:

$$
P V=n R T, \quad P \rightarrow 0, T \rightarrow \infty
$$

The agreement between a measured parameter, say pressure, and the corresponding one calculated with Eq 1, improves to the extent that pressure goes down and temperature goes up. This fact is shown in Eq 1 as $P \rightarrow 0$ and $T \rightarrow \infty$. This fact can also be observed from the calculation of $R$ with Eq $1(R=P V / n T)$. Thus many gases obey that equation within $0.1-1 \%$ at temperatures of approximately $25^{\circ} \mathrm{C}$ and pressures close to one atmosphere. This means that, at temperatures lower than $25^{\circ} \mathrm{C}$ and pressures higher than 1 atm results obtained with $\mathrm{Eq} 1$ for $R$ differ in a higher percentage relative to the accepted value for it ${ }^{6}$

Experience also shows that under same above limiting conditions, the pressure of a gas mixture is equal to the sum of the pressures that each gas would exert if it were alone in the container (Dalton's Law of Partial Pressures). This means that in the mixture each gas exerts the same pressure that it exerts when it is alone. From a molecular point of view, this implies that molecular volumes are extremely small as compared with the container volume and that molecules do no interact with each other so as to be detected by experiments.

Because each gas in the mixture under the limiting temperature and pressure conditions above obeys Eq 1, the equation of state for the whole mixture is:

$$
(P V)_{\text {lim }}=\left(\sum_{i} n_{i}\right) R T=\left(\sum_{i} x_{i}\right) n_{\text {tot }} R T, \quad P \rightarrow 0, T \rightarrow \infty, \text { any } x_{i}
$$

The above equation is Eq 1' at Table 1, $x_{i}$ is the molar fraction of the ith component in the mixture, $n_{i}$ is its mole number, $n_{\text {tot }}$ is total number of mole, and $P$ is total pressure. The pressure due to component $i, P$, relates to the total pressure as $P_{i}=x P$.

The above equation, and any other derived in this paper, applies to pure gases; "mixtures of one component". Therefore, we define a limiting real gas, pure or gas mixture, as one obeying Eq 1'. Thus, Eq 1' is the equation of state of limiting real gases, whether pure or gas mixture. The term real will be frequently omitted for brevity. Though limiting conditions are indicated for all equations in the first column of Table 1, the subscript lim is kept to emphasize column comparison. All equations are represented by symbol $E_{\lim }$ given at the end of this column.

The Chemical Potential of Limiting Real Gases. Let's assume the solid line at Fig. 1 depicts the chemical potential behavior with pressure, of a gas $i$ according to some general equation.

Let's then find out how that equation would look like at limiting physical conditions. For that purpose let's assume we have a limiting gas mixture $(P \rightarrow 0$ and $T \rightarrow \infty)$ in a container $A$ and in an identical one, $B$ (same volume), only pure gas $i$. Both containers are at the same temperature and contain the same number of moles of component $i$. According to Dalton's law the pressure of component $i$ is the same in both containers $(P \rightarrow 0)$. Let's also assume that these containers are separated by a rigid membrane, thermally conductive and permeable to gas $i$ only. The thermodynamics condition for phase equilibrium requires that $\mu_{i}^{A}=\mu_{i}^{B}$. For the pure gas we can write:

$$
\mu_{i}^{B}=\mu_{i}^{*}(T, P i)=\mu_{i}^{*}\left(T, x_{i} P\right)=\bar{G}_{, i} *\left(T, x_{i} P\right)
$$




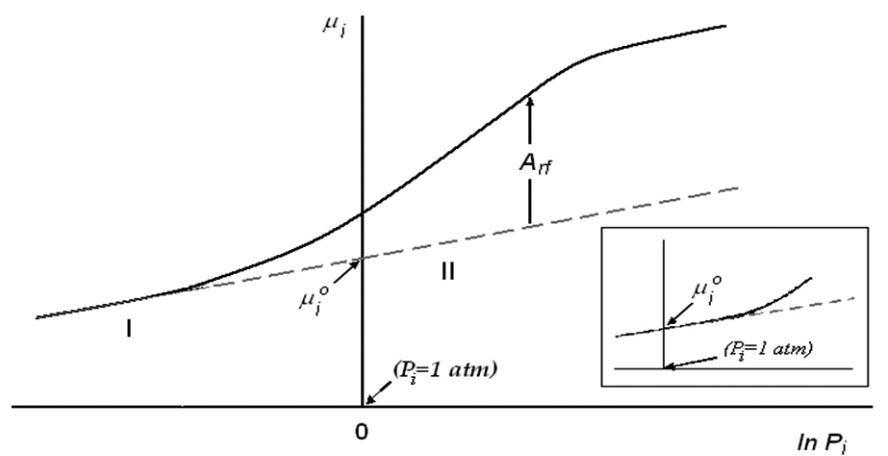

Figure 1. The chemical potential behaviour of a gas $i$. The solid line depicts the behaviour of a real gas at any $P_{i}$ value. The lowest part of the solid line describes the behaviour of a limiting gas; it is over straight line I. The dotted straight line -line I plus line II- shows the behaviour of a gas having the properties of a limiting gas at any $P$, value; the behaviour of a hypothetical gas (an ideal gas). $A_{r f}$ (the approximation-to-reality function) indicates how much the hypothetical (ideal) behavior of the gas must be corrected to match the real behavior. $\mu_{i}^{\circ}$ (or $\mu_{i}^{* \circ}$ in the text) is the chemical potential of a hypothetical standard state which occurs when the gas $i$ does not behave as a limiting gas at $1 \mathrm{~atm}$ pressure. $\mu_{i}^{\circ}$ in the inset is the chemical potential of a real standard state that occurs when the gas $i$ behaves as a limiting gas at $1 \mathrm{~atm}$ pressure.

The asterisk is for pure gas $i ; P_{i}$ is the partial pressure of component $i$, both pure and in the mixture; $x_{\text {in }}$ is the mole fraction of $i$ in the mixture; $P$ is total pressure of the mixture; and $\bar{G}^{*}$ is the mole Gibbs function of pure gas $i$. Thermodynamics also states that at constant temperature:

$$
d \mu_{i}^{*}\left(T, P_{i}\right)=d \bar{G}_{i}^{*}\left(T, P_{i}\right)=\bar{V}_{i}^{*}\left(T, P_{i}\right) d P_{i}=R T d \ln P_{i}
$$

where $\bar{V}_{i}$ is the mole volume of pure gas $i$. Let's consider the isothermal change of pure gas $i$ between states 1 and 2. The integration of the above differential between these states requires that gas $i$ accomplishes Eq 1' at any point in this interval and that it occurs over a straight line of $\mu_{*}^{*} v s \ln P$, as suggested by the differential equation. State 2 is the state with pressure $P_{i, 2}$ equal to the pressure of $i$ in the mixture, and temperature $T$. We want state 1 to be the standard state with pressure $P_{i,}=1 \mathrm{~atm}$ and the same temperature. While state 2 is a real state of gas $\left(P_{i,} \rightarrow 0\right)$, state 1 might be a hypothetical state (an ideal state) because the gas might not behave as a limiting real gas at the pressure of $1 \mathrm{~atm}$. Let's consider this possibility. The straight line satisfying all these conditions is drawn as a dotted line in Fig. 1; it is formed by lines I and II. Because line I matches exactly the lowest part of the solid line, it describes the behavior of the limiting gas. State 2 must be one point on this dotted line. Line II is an extrapolation of the limiting real gas behavior at pressures higher than those at line I. It therefore describes the behavior of a gas having the properties of a limiting gas at any pressure value, i.e. the behavior of a hypothetical gas (an ideal gas). The standard state is at the intercept of line II with $\mu_{\text {-axis, }}$ where pressure is by definition equal to $1 \mathrm{~atm}$. Hence, this standard state is a hypothetical state. Then, the integration of the differential equation above gives:

$$
\mu_{i}^{*}\left(T, P_{i, 2}\right)-\mu_{i}^{* \circ}(T)=R T \ln \left(P_{i, 2} / 1 \mathrm{~atm}\right)
$$

The superscript degree indicates the standard state of $i$ at a pressure of 1 atm. Because the standard state for $i$ necessarily implies pure $i$, the asterisk is redundant in $\mu_{i}^{* \circ}(T)$ and can be deleted: $\mu_{i}^{* \circ}(T)=\mu_{i}^{\circ}(T)$. Subscript 2 is also unnecessary and therefore it can be deleted as well: $\mu_{i}^{*}\left(T, P_{i, 2}\right)=\mu_{i}^{*}(T$, $P$.). Remember that $\mu_{i}^{*}(T, P i)=\mu_{i}^{B}$ and that $\mu_{i}^{B}=\mu_{i}^{A}$. Then by naming $\mu_{i}^{A}$ as $\mu_{i}$, we have $\mu_{i}^{*}(T, P i)=\mu_{i j i m}$. With all these changes and using symbol $P^{\circ}$ to represent the standard pressure of $1 \mathrm{~atm}$, the above equation becomes Eq 2'.

Equation 2' gives the chemical potential of component $i$ in the limiting mixture, $\mu_{i l i m}$, when the partial pressure of component $i$ is $P_{i}$, and the temperature and total pressure of the mixture are $T$ and $P$, respectively. $\mu_{i}^{o}(T)$ is the chemical potential for the standard state of component $i$, the latter being defined as pure gas $i$ at $1 \mathrm{~atm}$ and at temperature $T$ of the mixture, having the properties it would have at $P \rightarrow 0$. We say that Eq 2' is the expression for the chemical potential of limiting gases, pure or gas mixture.

Two points should be noted. First, as the solid line at Fig. 1 has been drawn arbitrarily with that shape, it might be of any other shape and besides appears under the dotted line. Second, if the departure of line II is at a pressure higher than one atmosphere, state 2 in above deduction, the standard chemical potential (the chemical potential at $1 \mathrm{~atm}$ ), $\mu_{i}^{\circ}(T)$, will be a point on line I, and, therefore, a real (limiting) state, as shown at the inset in Fig. 1.

In conclusion, Eq 2' is the equation for the lowest part of solid line in Fig 1 , where it becomes linear. It is the fundamental thermodynamics equation of limiting gases; all equations can be derived from it. Because of this, we alternatively define limiting gases as those obeying Eq 2'. This equation is not deduced in Physical Chemistry textbooks as we have done here (nor its representation as in Fig. 1), but, as mentioned earlier, it is derived from a general equation.

The Chemical Equilibrium Constant for Limiting Real Gases. All thermodynamics equations of limiting gases, pure or gas mixture can be derived from Eq 2'. For example, let's deduce the pressure-based chemical equilibrium constant, which is not deduced in textbooks as it is done here. The thermodynamics condition for chemical equilibrium requires that $\Sigma_{i} \mu_{i}=0$, where $\mu_{i}$ represents the chemical potentials of each participant in the chemical reaction and $v i$ their corresponding stoichiometric coefficients. For an all gasphase chemical reaction taking place at limiting pressures and temperatures, the application of Eq 2' to the equilibrium condition yields:

$$
\Sigma_{i} v_{i} \mu_{i}^{\circ}(T)+R T \Sigma_{i} v_{i} \ln \left(P_{i} / P^{\circ}\right)=\Sigma_{i} v_{i} \mu_{i}^{\circ}(T)+R T \ln \left[\Pi_{i}\left(P_{i} / P^{\circ}\right)^{v i}\right]=0,
$$

$P_{i}$ represents the partial equilibrium pressure of each participant in the reaction mixture. Because $\Sigma v \mu^{\circ}(T)$ is a constant value at fixed temperature, the product $\Pi_{i}(P / P)^{n i}$ in the logarithm must also be a constant value at fixed temperature. We name this constant $K_{\text {lim }}$ and define it as the chemical equilibrium constant for limiting gases. Let's represent an all gas-phase chemical equilibrium reaction as:

$$
a A+b B \leftrightarrow c C+d D
$$

Applying the above result to Eq 2 and omitting $\left(P^{\circ}\right)^{v i}$ for simplicity, Eq 3' is obtained. Sea)

3.2. Ideal Gases (generalizing limiting situations: the Fish in the Ideal

To get closer to the description of the complete real system we rule out the restrictions to the conclusions of the first part; we let parameters take all possible values in nature. However, in doing this we are inventing a fictional system, since we are taking our conclusions beyond the range where they are valid in reality. From a molecular point of view there are no molecular interactions and molecules are point masses in this system. In summary, we build up an ideal system of the same size as the real one but having the characteristics of the limiting reality. This is illustrated by Step II in Scheme 1.

Consistently with the idea above, gases in this ideal system accomplish all the equations of limiting gases but with no restrictions to their temperature, pressure and composition values. They are hypothetical or ideal gases. Their main equations are given in the second column in Table 1: Eq 4' is the equation of state of ideal gases, whether pure or gas mixture; Eq 5' is the expression for the chemical potential of ideal gases, pure or gas mixture; and Eq 6' is the chemical equilibrium constant for ideal gases. Thus, ideal equations are just extrapolations of their corresponding equations in the first column at any $P, T$, and $x_{i}$ values. This fact is represented by the equation at the end of the second column: $I=E$, where $I$ is for the ideal equation and the subscript lim is crossed out in the limit equation to indicate that limiting conditions have been ruled out.

Regardless of the above, a single equation is needed to define ideal gases from a thermodynamics point of view. We propose to choose Eq 5' because, as derived from Eq 2', it constitutes the fundamental thermodynamics equation of ideal gases. Therefore, we define ideal gases, pure or gas mixtures, as those obeying Eq 5'. Equation 5' is the equation for the dotted line in Fig. 1.

So, all equations in the second column in Table 1 are deduced (or imposed by definition) from their respective equations in the first column. Thus, we do not need to make new mathematical deductions. However, following the same reasoning used to deduce Eq 2' from Eq 1' and Eq 3' from Eq 2', Eq 5' can be deduced from Eq 4' and Eq 6' from Eq 5'. This is how textbooks currently deduce these equations.

It is necessary to highlight at this point that real gases exhibit ideal behavior only at limiting conditions and that, therefore, ideal gases do not exist; there is only ideal behavior of real gases. Ideal gases are a scientific invention. Consequently, and to avoid confusing students, the term limiting gases is used in this work to refer to real gases at limiting pressure and temperature while the term ideal gases refers to hypothetical gases behaving as limiting gases at any temperature and pressure. 


\subsection{General Real Gases (back to reality: the Fish in the Real Sea)}

From now on, the terms real and general real will be used as synonyms. General systems and equations will refer to systems existing or equations that are valid under any physical condition.

From Fiction to Reality: the $A_{r f}$ Function. All equations in the section above are general equations because they cover the idealized reality they have been invented for with no restrictions to their applicability. Next, it is necessary to see how those general equations are used to explain (or attempt to explain) the exact reality as a whole.

The strategy to accomplish the objective above is as follows. First, we make the parameters in the right side of the ideal expressions represent their values in the real system. Second, we modify these general ideal equations by functions that we assume contain all the relevant information about the reality ignored by the ideal system. As mentioned, we will name these functions the approximation-to-reality functions, $A_{v f}$ This scheme is represented by Step III in Scheme 1.

The connection of a real equation, $R$, to the ideal equation, $I$, is given by one of the following algorithms:

$$
\text { a) } R=I x \mathcal{A}_{r f} \text { or b) } R=I+\mathcal{A}_{r f}
$$

Equations 3 are Eqs 10' in Table 1. Because $I=E_{\text {timin' }}$ any real equation relates to its corresponding limit equation as follows:

$$
\text { a) } R=E_{\text {fim }} \times \mathcal{A}_{r f} \text { or b) } R=\mathcal{E}_{\text {fim }}+\mathcal{A}_{r f}
$$

$\mathcal{A}_{r f}$ functions are constructed so that real equations can keep the mathematical forms of their corresponding ideal equations which, in turn, have the form of their corresponding limit equations. The mathematical form of each ideal equation will determine the use of either algorithm 3a or 3b. Keeping the same mathematical form will allow profiting from all results obtained with previous equations and, mainly, making better comparisons among them. Besides, the mathematical forms of ideal equations are so simple that it is timesaving to keep them. While textbooks follow a strategy different to the one of this paper, they resort to the same criterion to derive the mathematical form of the real equations from the ideal equations.

According to the above framework, real gases are to accomplish all equations of ideal gases but be modified by appropriate $\mathcal{A}_{r f}$ functions. Their main equations are given in the third column in Table 1; they are modifications of their corresponding ideal equations in the second column. The corresponding $\mathcal{A}_{\text {rf }}$ functions are given in the fourth column in Table 1. While some Physical Chemistry textbooks make some relationships between ideal equations and real equations explicit ${ }^{9}$, they remain only implicit in most texts $5,7,8$. Anyway, all equations follow the general pattern shown by Eqs 10' in Table 1, Eqs 3 above, no matter how each author arrives to them. Nevertheless and by the reasons given, textbooks do not explicit this pattern in some general formalism (equations) as we have done it here.

Consistently with the above picture, we propose to choose Eq 8' to define real gases from a thermodynamics point of view; as derived from Eq 5', Eq $8^{\prime}$ is the fundamental thermodynamics equation of real gases. Therefore, we define real gases, pure or gas mixtures, as those obeying Eq 8'.

The way real equations are normally obtained is by replacing the limiting experimental parameter in ideal equations by a new one that will correct ideality ${ }^{5,7-9}$. For example, we replace $P_{i}$ in Eq $5^{\prime}$ by the parameter known as fugacity, $f_{i}$, which relates to the former by equation $\chi_{i}=f_{i} / P_{i}, \chi_{i}$ being the fugacity coefficient. The same procedure is followed for condensed phases, where the limiting parameter, $x_{i}$ (mole fraction), is replaced by the parameter known as activity, $a$, in the respective ideal equation (whose form is the same as Eq 5'). Both parameters are connected by equation $\gamma_{i}=a_{i} / x_{i} ; \gamma_{i}$ is known as the activity coefficient. Thus, as mentioned before, the current procedure also constructs equations having the same mathematical forms as their corresponding ideal equations. However, we believe that the approximationto-reality function concept introduces the corrections to ideality in a more intuitive and systematic way; not as particular solutions for each case. Finally, approximation functions allow introducing first-year students to the concept of real equations without discussing concepts like fugacities, activities, and others.

Parameters like $\chi_{i}$ and $\gamma_{i}$ are commonly referred to as measures of the deviation from ideality. To our opinion, this concept might confuse students because reality is presented as a deviation from ideality. In our context, it seems more appropriate to refer to functions $A$ as measures of the approximation to reality, because that is our purpose when introducing them.

Thus, the need of correction functions $\left(A_{r p} \gamma, \chi\right.$, etc $)$ arises from physical arguments but their mathematical forms arise from mathematical convenience. It is necessary to use Thermodynamics to formulate these functions, and, within our framework, this is what we will do in the following sections. However, it is a task for experimentalists to evaluate these functions from measurable properties and for theorists to search their meanings and formulations from the microscopic properties of matter. Much work has been done in this regard.

The Equation of State of Real Gases. Pressure exerted by molecules and volume available to them change in going from ideal gases to real ones. From a molecular viewpoint, this means that molecular volumes and molecular interactions affect these parameters in the extent the system moves away from limiting physical conditions and therefore from the boundary conditions imposed to ideal gases. We correct both parameters simultaneously by applying algorithm $3 \mathrm{a}$ to the ideal equation, Eq 4'. Then the product of real volume and pressure is as follows:

$(P V)_{\text {real }}=(P V)_{\text {ideal }} \cdot A_{r f}=\left(\Sigma_{i} n_{j}\right) R T \cdot A_{r f}=\left(\Sigma_{i} x_{i}\right) n_{\text {tot }} R T \cdot A_{r f}$, any $T, P$ and $x_{i}$

Equation 4 is the explicit form of Eq 7', where $A_{r f}=A_{r f}\left(T, P, x_{i}\right)$. Examples of appropriate approximation functions are the virial equations of states. Equation 11' is an example of a virial equation for a pure gas. This equation and a similar one that is a function of the molar volume of the gas are given elsewhere ${ }^{5,7,8}$. Equation 4 is the equation of state for real gases, whether pure or gas mixture, at any temperature, pressure, and composition.

Equation 1' represents a limiting but real situation. Therefore, under our scheme it must be considered as a particular solution of general Eq 4. Hence, we must have that:

$$
\underset{P i \rightarrow 0, T \rightarrow \infty}{\operatorname{limit}} A_{r f}=1
$$

As required, all virial equations satisfy the above equation (see Eq 11').

The Chemical Potential of Real Gases. As mentioned above, Eq 8' is the expression for the chemical potential of real gases. It results from applying algorithm $3 \mathrm{~b}$ to $\mathrm{Eq} 5$ '. Let's make this result more explicit:

$$
\mu_{i, \text { real }}=\mu_{i}^{o}(T)+R T \ln \left(P_{i} / P^{o}\right)+A_{r f}, \quad \text { any } T \text { and } P_{i}
$$

$A_{v f}=A_{v f}\left(T, P, x_{j}\right)$. As in Eq 5', $\mathrm{P}_{\mathrm{i}}$ in above equation is the pressure of component $\mathrm{i}$ in the mixture. However, for the same reasons above, $\mathrm{P}_{\mathrm{i}}$ in this equation is not necessarily equal to the pressure component $i$ would have if it were alone. Also, as in $\mathrm{Eq} 5^{\prime}, \mu^{\circ}(\mathrm{T})$ is the chemical potential for the standard state of component $\mathrm{i}$ in the gas mixture, this state being the pure gas $i$ at $1 \mathrm{~atm}$ and temperature $\mathrm{T}$ of the mixture, behaving as an ideal gas. Therefore, if the real gas does not behave ideally at $1 \mathrm{~atm}, \mu_{\mathrm{i}}^{\mathrm{o}}(\mathrm{T})$ is the chemical potential of a fictional state of the gas. All corrections to ideality are contained in the reality function, $\mathrm{A}_{\mathrm{rf}}$.

Equation 5 is the equation for the solid line in Fig. 1. As mentioned before, this solid line could be over or under the dotted line representing the ideal behavior of gas i. For the latter case the arrow indicating $A_{r f}$ would point down. Since $A_{r f}$ indicates how much the hypothetical (ideal) behavior of the gas must be corrected to match the real behavior, we will refer to the arrow up as the measure of the positive approximation to reality, or, in opposite sense, as the negative deviation from reality; while for the arrow down we will refer to it as the measure of the negative approximation to reality, or, in opposite sense, as the positive deviation from reality.

Equation 2' is for limiting real gases. Therefore, it must be a particular case of general Eq 5. Hence:

$$
\operatorname{limit}_{P i \rightarrow 0, T \rightarrow \infty} A_{r f}=0
$$

Under this condition, Eq 5 becomes Eq 2'; Eq 5 is now describing the lowest part of the solid line in Fig 1.

The choice of algorithm $3 \mathrm{~b}$ requires that the reality function be defined as given by Eq 12'. As already mentioned, $\chi_{i}$ is the fugacity coefficient of component $i$ in the gas mixture, a dimensionless parameter; and $f_{i}$ is the fugacity of component $i$ in the gas mixture, having pressure units. $f_{i}=f_{i}(P, T, x)$. By replacing Eq 12 ' in Eq 5, we obtain:

$$
\mu_{i, \text { real }}=\mu_{i}^{o}(T)+R T \ln \left(f_{i} / P^{o}\right), \quad \text { any } T \text { and } P_{i}
$$

As stated before, we have obtained an equation of the same mathematical form as the ideal equation (Eq 5') was obtained; fugacity plays the same role as pressure in the ideal equation. Equation 6 is the explicit formulation of the 
fundamental thermodynamics equation for real gases, expressed by either Eq 5 or Eq 8 ,

Because general Eqs 5 and 6 must give account of the ideal and limiting behavior of gases, Eqs 5' and 2', respectively, it follows that $\chi_{i}=1$ for ideal and limiting gases. Note also, that $\chi_{i}=1$ for the standard state. Fugacity coefficient values for other situations can be assessed from measurable gas properties ${ }^{5,7-9}$.

The Chemical Equilibrium Constant for Real Gases. Equation 9' is the equation of chemical equilibrium constant for real gases. It arises from applying algorithm 3a to Eq 6'. Let's make this result explicit:

$$
K_{\text {real }}=\left(P_{C}^{c} P_{D}^{d} / P_{A}^{a} P_{B}^{b}\right) \cdot A_{r f}, \quad \text { any } T, P_{i}
$$

Equation 3' is for limiting gases. Therefore, it must be a particular case of general Eq 7. This demands that:

$$
\underset{P i \rightarrow 0, T \rightarrow \infty}{\operatorname{limit}} A_{r f}=1
$$

The choice of algorithm 3a requires that the reality function be defined as:

$$
A_{r f}=\Pi_{i} \chi_{i}^{n i}=\Pi_{i}\left(f_{i} / P_{i}\right)^{n i}, \text { with } \chi_{i}=f_{i} / P_{i}, \text { any } T \text { and } P_{i}=x_{i} P
$$

By definition, $\chi_{\mathrm{i}}$ 's are the same as above. $v_{\mathrm{i}}$ represents the stoichiometric coefficients of each participant in the chemical reaction. Applying the above equation to a reaction as represented by Eq 2, Eq 13' is obtained. Replacing this result in Eq 7 yields:

$$
K_{\text {real }}=f_{C}^{c} f_{D}^{d} / f_{A}^{a} f_{B}^{b}, \quad \text { any } T \text { and } P_{i}
$$

Equation 8 is the explicit form of Eq 9'. As expected, this general equation is of the same form as ideal Eq 6'. Because this general equation must become ideal Eq 6' and limit Eq 3' at their respective boundary conditions, $\chi_{i}=1$ for ideal and limiting gases, as deduced in the above section.

Equation 8 could have been obtained from Eq 6 following same reasoning we used to deduce Eq 3' from Eq 2'. This is the current way Eq 8 is deduced in textbooks. Another alternative is just substituting $P_{i}$ by $f_{i}$ in Eq 6'; as concluded from Eq 6, fugacity in the real equation plays the same role as pressure does in the ideal equation. However, we have used the described procedure to follow the logics of the presentation.

\subsection{The Liquid-Phase Solutions}

Liquid-phase system analysis follows exactly the same strategy used for gases in this work, showing equivalent results. Two solution models (ideal models) can be constructed, one where all solution species are equally treated and another where a distinction is made between solutes and solvent. The first model corresponds to the ideal solutions and the second model to the ideal dilute solutions, as defined elsewhere ${ }^{7-9}$. As for the ideal gas system, these ideal solution systems are generalizations of limiting real solutions. These limiting real solutions (simplified experimental systems or experimental models) are now "the fish in the bowl". As for gases the equation of state is the starting limit equation, for the first solution system the starting limit equation is Raoult's law, while for the second solution system are Raoult's and Henry's laws together. For briefness this analysis is not presented here.

The search of general equations without resorting to equations for particular cases but to general situations and using general thermodynamics formalisms has also aimed some articles. Thus, general equations have been developed, whose validity is demonstrated in all cases by applying them to limiting situations, for which results are known ${ }^{4}$, or by arriving to accepted equations for ideal systems ${ }^{11}$. This last situation has also been commented in another article $^{10}$. Because those methodologies require advanced knowledge of thermodynamics, they do not allow illustrating to first-year chemistry students the various stages of learning of the discipline. By contrast, our methodology moves in the reverse direction, allowing us to follow the evolution of an equation from its most elementary form (under limiting conditions, a particular equation), learned at General Chemistry courses, to its most complex forms (under general conditions, a general equation) learned at Physical Chemistry courses. In a direction similar to our work, a reasoning scheme and related equations have been developed to teach the state function entropy, which start with simple and empirically-supported equations to finish with the fundamental equations of entropy ${ }^{12}$. According to the authors, this learning strategy should be more intuitive to students and easier to understand than the classical method of teaching this concept, which the authors describe as a tortuous chain of reasoning. Nevertheless, it is not the purpose of this work correlate limit equations with their corresponding general equations nor is intended for the teaching of students at introductory chemistry courses, which are, among others, the objectives of our work. On another hand, a rubber-elastic material, different to solids, liquids and gases commonly used in teaching Thermodynamics, has been proposed elsewhere ${ }^{10}$ to teach the discipline at introductory level. The author derives equations for this system by making analogies with ideal gases. In this regard, the article does not transit between a limit equation and its generalized formulation, as we do, but it remains in one particular situation, the ideal situation. In a general sense, science education literature is abundant in articles dealing with conceptual and reasoning difficulties that students and instructors encounter when learning or teaching chemistry, respectively. They refer to specific subjects (a particular compound, reaction or system) ) $^{13,14}$ or to fundamental concepts at macroscopic and microscopic level, as for instance, concepts in thermodynamics ${ }^{6,15,16}$ and in matter structure ${ }^{17,18}$, respectively. Some articles give specific instructions on how to correct misconceptions or how to clarify complex subjects. In two critical cases, one article suggests that a substantial review of teaching strategies regarding colligative properties is needed ${ }^{19}$, while another article proposes a new methodology to teach the structure of matter ${ }^{17}$. Only in this last case a holistic overview of a problem, as the one presented in this work, is given; authors recommend teaching chemistry progressively, and describe a modern way starting with observations at a macroscopic level, interpreting these at an atomic and molecular level, and then at an electronic and nuclear level.

\section{CONCLUSIONS}

Limit equations play a crucial role as the starting point for understanding reality as a whole; by ruling out their restrictions and making use of appropriate functions they become equations of general validity; Scheme 1

Based on above, we have developed an alternative method of presenting fundamental chemical thermodynamics equations. Thus, by using the equation of state for gases under limiting physical conditions, we have deduced the fundamental thermodynamics equations for limiting real gases. Then, by ruling out the restrictions for these limit equations, we have obtained equations for idealized gases (ideal or hypothetical gases). Finally, by making use of appropriate functions we have converted these ideal general equations into equations for real gases at any physical conditions (Eqns 3a); real general equations. We believe the strategy of developing the equations strictly under Scheme I and the consequent routes followed in this work to construct Table 1 , should result more intuitive and logical to students and therefore be more didactical than the current one.

Scheme 1 and Table 1 omitting Eq 2' would allow instructors to tell general chemistry students what part of chemistry they are learning at present, not going at this stage into the mathematical details of this text but just explaining to them the general relationship existing between the limit equations they are beginning to learn and the general ones they will learn in future courses. In this regard it is necessary to emphasize, that General Chemistry instructors should merely present the limiting cases omitting Eq 2 and then warn students that more accurate equations will be encountered in future courses.

Students at introductory physical chemistry courses would know that courses they are beginning to attend will teach how to connect, still in a general ways, what they have learned previously - the limit equations - with what they will learn now and later - ideal equations and general real equations-, respectively. And that the latter will be done by discovering the functions changing ideal equations into real ones. Besides, they would be told about the necessity of theoretical disciplines as quantum chemistry and statistical mechanics, to discover the meaning of the terms contained into those transforming functions. Finally, students at upper physical chemistry courses could examine the paper on their own as a recapitulation exercise. We believe that explaining the several steps of their chemistry learning (introductory, medium and upper level) to students is something pedagogically meaningful.

Finally, we have introduced terms that we believe should help clarifying to students some concepts that might result misleading, such as limiting gases to distinguish them from ideal gases; ideal behavior of real gases from being ideal gases; approximation to reality instead of deviation from ideality.

\section{ACKNOWLEDGMENTS}

Authors wish to thank Professor Ana Tejeda, from the Faculty of Humanities at the Universidad Católica del Norte, for her helpful assistance and suggestions in the edition of this article. Authors also thank the financial support from DGIP/UCN. 
Note

1. A strategy different to the one given by Eq 7' involves modifying each term of the ideal gas equation on the basis of theoretical considerations. A wellknown example is van der Waals equation for pure gases: $\left(P+a n^{2} / V^{2}\right)(V-n b)$ $=n R T$, where $a$ and $b$ are different constants for different gases and $P$ and $V$ are the experimental pressure and volume values.

\section{REFERENCES}

1. C.E. Mortimer, Chemistry, $5^{\text {th }}$ ed., Wadsworth, Belmont, $C A, 1983$

2. R.H. Petrucci, W.S. Harwood, General Chemistry: Principle and Modern Applications, $7^{\text {th }}$ ed., Prentice Hall, New Jersey, 1997.

3. R. Chang, Chemistry, $6^{\text {th }}$ ed., McGraw-Hill, Boston MA, 1998.

4. V. Talanquer, "Reclaiming the Central Role of Equations of State in Thermodynamics", J. Chem. Educ. 83, 127, (2006)

5. G.N. Lewis, M. Randall, Thermodynamics, revised by K.S. Pitzer and L. Brewer, $2^{\text {nd }}$ ed., McGraw-Hill, New York, 1961.

6. C. Contreras-Ortega, N. Bustamante, J. L. Guevara, C. Portillo, V. Kesternich, "The Physical Meaning of the Mathematical Formalism Present in Limiting Chemical Equations; Or, How Dilute Is Dilute? $J$. Chem. Educ. 84, 788, (2007)

7. I.N. Levine, Physical Chemistry, $5^{\text {th }}$ ed., McGraw-Hill College, New York, 2001.

8. P.W. Atkins, J. de Paula, Physical Chemistry, $7^{\text {th }}$ ed., W.H. Freeman \& Company, New York, 2001.
9. G.W. Castellan, Physical Chemistry, $3^{\text {rd }}$ ed., Narosa, Massachussets, 1995.

10. B. Smith, "Using Rubber-Elastic Material-Ideal Gas Analogies to Teach Introductory Thermodynamics. Part I: Equations of State", J. Chem. Educ. 79, 1444, (2002)

11. T. J. Leo, P. Pérez-del-Notario, "A Generalized Deduction of the IdealSolution Model". J. Chem. Educ. 83, 145, (2006)

12. K. S. Sanchez, R. A. vergenzi, "Understanding Entropy: Using the Fundamental Stability Conditions". J. Chem. Educ. 71, 562 (1994)

13. B. M. Naah, M. J. Sanger, "Student Misconceptions in Writing Balanced Equations for Dissolving Ionic Compounds in Water", Chem. Educ. Res. Pract. 13, 186, (2012)

14. A. Boudreaux, C. Campbell, "Student Understanding of Liquid-Vapor Phase Equilibrium", J. Chem. Educ. 89, 707, (2012)

15. M. F. Granville, "Student Misconceptions in Thermodynamics", J. Chem. Educ. 62, 847, (1985)

16. A. Ben-Naim, "Entropy: Order or Information", J. Chem. Educ. 88, 594, (2011)

17. P. G. Nelson, "Teaching Chemistry Progressively: From Substances, to Atoms and Molecules, to Electrons and Nuclei", Chem. Educ. Res. Pract. 3, 215, (2002)

18. H. Özmen, "The Influence of Computer-Assisted Instruction on Students' Conceptual Understanding of Chemical Bonding and Attitude Toward Chemistry: A Case for Turkey", Computers \& Education, 51, 423, (2008)

19. T. Pinarbasi, M. Sozbilir, N. Canpolat, "Prospective Chemistry Teachers' Misconceptions about Colligative Properties: Boiling Point Elevation and Freezing Point Depression”, Chem. Educ. Res. Pract. 10, 273, (2009) 